\title{
Mathematical Analysis on Heat Transfer during Peristaltic Pumping of Fractional Second-Grade Fluid through a Nonuniform Permeable Tube
}

\author{
Siddharth Shankar Bhatt, ${ }^{1}$ Amit Medhavi, ${ }^{2}$ and R. S. Gupta ${ }^{1}$ \\ ${ }^{1}$ Department of Applied Sciences and Humanities, Kamla Nehru Institute of Technology, Sultanpur, Uttar Pradesh 228118, India \\ ${ }^{2}$ Department of Mechanical Engineering, Kamla Nehru Institute of Technology, Sultanpur, India
}

Correspondence should be addressed to Siddharth Shankar Bhatt; shankarbhatt56@gmail.com

Received 8 March 2016; Accepted 19 May 2016

Academic Editor: Jose M. Montanero

Copyright (C) 2016 Siddharth Shankar Bhatt et al. This is an open access article distributed under the Creative Commons Attribution License, which permits unrestricted use, distribution, and reproduction in any medium, provided the original work is properly cited.

\begin{abstract}
This mathematical study is related to heat transfer under peristaltic flow of fractional second-grade fluid through nonuniform cylindrical tube with permeable walls. The analysis is performed under low Reynolds number and long wavelength approximation. The analytical solution for pressure gradient, friction force, and temperature field is obtained. The effects of appropriate parameters such as Grashof number, nonuniformity of tube, permeability of tube wall, heat source/sink parameter, material constant, fractional time derivative parameter and amplitude ratio on pressure rise, friction force, and temperature distribution are discussed. It is found that an increase in amplitude ratio and material constant causes increase in pressure but increase in nonuniformity of the tube causes decrease in pressure. It is also observed that variation of friction force against flow rate shows opposite behavior to that of pressure. Increase in temperature is also observed due to increase in heat source/sink parameter at inlet as well as downstream.
\end{abstract}

\section{Introduction}

The word "peristalsis" originated from the Greek word "peristaltikos," which means clasping and compressing. Peristalsis is a mechanism of fluid transport through deformable vessels with the aid of a progressive contraction/expansion wave along the vessel. It is an important mechanism of fluid transport in different parts of the entire physiological system. Peristaltic flow appears in urine transport from kidney to bladder, the movement of spermatozoa in the ductus efferentes of the male reproductive tract and in the vasomotion of small blood vessels such as arterioles, venules, and capillaries. Peristaltic motion finds application in industry such as heart lung machine and roller pump. Peristaltic motion was first studied clinically in an article given by Bayliss and Starling [1] and much later Latham [2] theoretically investigated peristalsis using fluid mechanics principles. The work of Jaffrin and Shapiro [3] throws light on various parameters involved in the analysis of peristaltic pumping. Kumar et al. [4] studied unsteady peristaltic pumping in finite length tube with permeable wall.
Interaction of peristalsis with heat transfer plays an important role in biomedical science. Models of microvascular heat transfer are being increasingly used for optimizing thermal therapies such as hyperthermia treatment. Thermodynamic aspects of blood become significant in processes like oxygenation and hemodialysis. Victor and Shah [5] studied heat transfer to blood flowing in a tube. Srinivas and Kothandapani [6] investigated peristaltic transport in an asymmetric channel with heat transfer. Muthuraj and Srinivas [7] studied mixed convective heat and mass transfer in a vertical wavy channel with traveling thermal waves and porous medium. Lots of investigations have been done for uniform channel or tube; however, most physiological vessels, for example, ureters, esophagus, intestine, and ductus efferentes of the reproductive tract, possess nonuniform geometries. Some researcher have used nonuniform geometry for analysis such as Radhakrishnamacharya and Radhakrishna [8] who discussed heat transfer to peristaltic transport in a nonuniform channel. Ellahi et al. [9] discussed effect of heat and mass transfer on peristaltic flow in a nonuniform rectangular duct. However, the study of heat flow 
during peristalsis has not been given much consideration by investigators.

Most physiological fluids exhibit both viscous and elastic properties. Viscoelastic models are derived by using classical mechanics laws, that is, Newton's law for viscous liquids and Hooke's law for elastic solids. In modern days, fractional calculus is a rapidly growing field of research in physics, biology, and medical engineering. By using fractional calculus, the viscoelastic behavior of fluid can be successfully explained. Fractional second-grade calculus operator is actually a generalization to deal with integrals and derivatives of noninteger order. Fractional second-grade model can be obtained by replacing ordinary time derivative to fractional time derivative. This model is applied to the study of movement of chyme through small intestine, esophagus, and so forth. Using the concept of fractional calculus some visoelastic models have been developed such as fractional Maxwell, fractional Zener, fractional anti-Zener or fractional Jeffrey, and fractional Burgers' models. Number of researchers has studied unsteady flows of viscoelastic fluids using different models like fractional Maxwell model, fractional second-grade fluid model, fractional Burgers' model and fractional generalized Burgers' model, and fractional Oldroyed-B model in channels, annulus, or tubes. Qi and $\mathrm{Xu}$ [10] studied unsteady flow of viscoelastic fluid with fractional Maxwell model in a channel. Tripathi [11] explored the transportation of a viscoelastic fluid with fractional second-grade model by peristalsis through cylindrical tube under the assumptions of long wavelength and low Reynolds number. Tripathi and Bég [12] studied peristaltic propulsion of generalized Burgers' fluid through a nonuniform porous medium with chyme dynamics through diseased intestine. Hameed et al. [13] analysed the heat transfer on peristaltic flow of the fractional second-grade fluid confined in a uniform cylindrical tube in presence of magnetic field. Rathod and Tuljappa [14] have studied peristaltic flow of fractional second-grade fluid through a cylindrical tube with heat transfer under the assumption of long wavelength and low Reynolds number assumption.

The objective of this study is to investigate the effect of various concerned parameters on fractional secondgrade fluid with heat transfer and peristaltic flow through nonuniform tube with permeable walls. The effects of these parameters have been studied under long wave length and the low Reynolds number approximation. The problem is solved analytically by the use of fractional calculus. The obtained expressions are utilized to discuss the influences of various physical parameters.

\section{Basic Definitions}

Definition 1. The Riemann-Liouville fractional integral operator of order $\alpha>0$ of a function $f(x):(0, \infty) \rightarrow R$ is given by [15]

$$
f(x)=\frac{1}{\Gamma(\alpha)} \int_{0}^{x}(x-\xi)^{\alpha-1} f(\xi) d \xi, \quad \alpha>0, x>0 .
$$

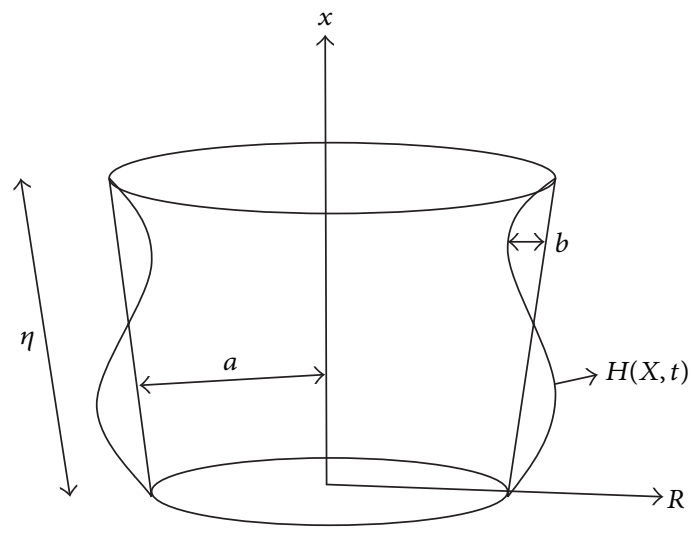

FIGURE 1: Geometry of the problem.

Definition 2. The fractional derivative of order $\alpha>0$ of a continuous function $f(x):(0, \infty) \rightarrow R$ is given by [15]

$$
\begin{aligned}
& D^{\alpha} f(x)=\frac{1}{\Gamma(m-\alpha)}\left(\frac{d}{d x}\right)^{m} \int_{0}^{x}(x-\xi)^{\alpha-1} f(\xi) d \xi \\
& \text { for } m-1<\alpha \leq m, m \in N, x>0, f \in C_{-1}^{m},
\end{aligned}
$$

where $m=[\alpha]+1$, provided that right-hand side is point-wise defined on $(0, \infty)$.

Remark 3. For example, $f(x)=x^{\beta}$; we quote for $\beta>-1$; in (2) one can get

$$
D^{\alpha} x^{\beta}=\frac{\Gamma(\beta+1)}{\Gamma(\beta-\alpha+1)} x^{\beta-\alpha}
$$

giving in particular $D^{\alpha} x^{\beta-n}=0, n=1,2,3, \ldots, N$, where $N$ is the smallest integer greater than or equal to $\alpha$.

\section{Mathematical Modelling}

Consider the flow of an incompressible fractional secondgrade fluid (as shown in Figure 1) due to peristaltic transport induced by sinusoidal wave trains propagating with constant speed $c$. The temperature of walls of tube is $T_{0}$. The constitutive equation for viscoelastic fluid with fractional secondgrade model is given by

$$
\widetilde{s}=\mu\left(1+\tilde{\lambda}_{1}^{\alpha} \frac{\partial^{\alpha}}{\partial \tilde{t}^{\alpha}}\right) \dot{\gamma},
$$

where $\tilde{t}, \widetilde{s}, \dot{\gamma}$, and $\tilde{\lambda}_{1}$ is time, shear stress, rate of shear strain, and material constant, respectively, $\mu$ is viscosity, and $\alpha$ is fractional time derivative parameters such that $0<\alpha \leq 1$. This model reduces to second-grade models when $\alpha=1$ and classical Navier-Stokes model is obtained by substituting $\tilde{\lambda}_{1}=0$.

The geometry of wall surface is given by

$$
H=a+B^{\prime} X+b \sin \frac{2 \pi}{\eta}(X-c t),
$$


where $a, b, \eta, c$, and $t$ are radius of the tube at inlet, wave amplitude, wave length, wave propagation speed, and time, respectively. $B^{\prime}$ is a constant whose magnitude depends on the length of tube.

The governing equations of the motion of viscoelastic fluid with fractional second-grade model through inclined tube for axisymmetric flow are given by

continuity equation:

$$
\frac{1}{R} \frac{\partial(R V)}{\partial R}+\frac{\partial U}{\partial X}=0
$$

momentum equation:

$$
\begin{aligned}
\rho( & \left.\frac{\partial U}{\partial t}+U \frac{\partial U}{\partial X}+V \frac{\partial U}{\partial R}\right) \\
= & -\frac{\partial p}{\partial X} \\
& +\mu\left(1+\tilde{\lambda}_{1}^{\alpha} \frac{\partial^{\alpha}}{\partial \tilde{t}^{\alpha}}\right)\left[\frac{1}{R} \frac{\partial(R(\partial U / \partial R))}{\partial R}+\frac{\partial^{2} U}{\partial X^{2}}\right] \\
& +\rho g \alpha_{1}\left(T-T_{0}\right), \\
\left(\frac{\partial V}{\partial t}+U \frac{\partial V}{\partial X}+V \frac{\partial V}{\partial R}\right) & \frac{\partial p}{\partial R}+\mu\left(1+\tilde{\lambda}_{1}^{\alpha} \frac{\partial^{\alpha}}{\partial \widetilde{t}^{\alpha}}\right)\left[\frac{1}{R} \frac{\partial(R V)}{\partial R}+\frac{\partial^{2} V}{\partial X^{2}}\right]
\end{aligned}
$$

energy equation:

$$
\begin{aligned}
& \rho c_{p}\left(\frac{\partial T}{\partial t}+U \frac{\partial T}{\partial X}+V \frac{\partial T}{\partial R}\right) \\
& =K\left(\frac{\partial^{2} T}{\partial R^{2}}+\frac{1}{R} \frac{\partial T}{\partial R}+\frac{\partial^{2} T}{\partial X^{2}}\right)+Q_{0}
\end{aligned}
$$

We introduce nondimensional parameters

$$
\begin{aligned}
V^{\prime} & =\frac{V}{c \delta}, \\
U^{\prime} & =\frac{U}{c}, \\
X^{\prime} & =\frac{X}{\eta}, \\
R^{\prime} & =\frac{R}{a}, \\
\lambda^{\prime} & =\frac{\tilde{\lambda}_{1} c}{\eta},
\end{aligned}
$$

$$
\begin{aligned}
\delta & =\frac{a}{\eta}, \\
p^{\prime} & =\frac{p a^{2}}{\mu c \eta}, \\
\operatorname{Re} & =\frac{\rho a c \delta}{\mu}, \\
\theta & =\frac{T-T_{0}}{T_{0}}, \\
\operatorname{Pr} & =\frac{\mu c_{p}}{K}, \\
\mathrm{Gr} & =\frac{\rho g \alpha_{1} a^{2} T_{0}}{\mu c}, \\
t^{\prime} & =\frac{c t}{\eta}, \\
\beta & =\frac{a^{2} Q_{0}}{K T_{0}}, \\
h & =\frac{H}{a},
\end{aligned}
$$

where $\rho$ is density of fluid, $Q_{0}$ is the constant heat, and $p, U, V, R, \mu, K, \alpha_{1}, \operatorname{Pr}, \beta$, and $\mathrm{Gr}$ stand for pressure, axial velocity, radial velocity, radial coordinate, coefficient of viscosity, thermal conductivity, coefficient of expansion, Prandtl number, source/sink parameter, Grashof number, respectively.

Using nondimensional parameters given in (9) and applying long wavelength and low Reynolds number approximation, (7) and (8) reduce to (after dropping primes)

$$
\begin{aligned}
\frac{\partial p}{\partial X}= & \left(1+\lambda^{\alpha} \frac{\partial^{\alpha}}{\partial t^{\alpha}}\right)\left[\frac{1}{R} \frac{\partial(R(\partial U / \partial R))}{\partial R}\right] \\
& +\theta \mathrm{Gr}, \\
\frac{\partial p}{\partial R}= & 0, \\
\frac{\partial^{2} \theta}{\partial R^{2}}+\frac{1}{R} \frac{\partial \theta}{\partial R}+\beta & =0 .
\end{aligned}
$$

The boundary conditions in dimensionless form are given as follows:

$$
\begin{aligned}
& \frac{\partial \theta}{\partial R}=0 \quad \text { at } R=0, \theta=0 \text { at } R=h, \\
& \frac{\partial U}{\partial R}=0 \quad \text { at } R=0, U=-k \frac{\partial U}{\partial R} \text { at } R=h,
\end{aligned}
$$

where $k$ is the slip parameter including slip [16].

The nondimensional wall surface geometry, $h$, is given as

$$
h=1+B X+\emptyset \sin 2 \pi(X-t),
$$

where $B=B^{\prime} \eta / a, \emptyset=b / a$ is amplitude ratio. 
On solving (10) with boundary condition (11), the following is obtained:

$$
\begin{aligned}
(1+ & \left.\lambda^{\alpha} \frac{\partial^{\alpha}}{\partial t^{\alpha}}\right) U \\
= & \left(\frac{R^{2}}{4}-\frac{h^{2}}{4}-\frac{k h}{2}\right) \frac{\partial p}{\partial X} \\
& \quad-\frac{G r \beta}{64}\left(4 R^{2} h^{2}-R^{4}-3 h^{4}-4 k h^{3}\right), \\
\theta= & \frac{\beta}{4}\left(h^{2}-R^{2}\right) .
\end{aligned}
$$

The volumetric flow rate is calculated as

$$
\bar{Q}=\int_{0}^{h} 2 \pi R U d R
$$

Using (13) in (15), the following is obtained:

$$
\begin{aligned}
\left(1+\lambda^{\alpha} \frac{\partial^{\alpha}}{\partial t^{\alpha}}\right) \frac{\bar{Q}}{\pi}= & \left(-\frac{h^{4}}{8}-\frac{k h^{3}}{2}\right) \frac{\partial p}{\partial X}+\frac{4 \mathrm{Gr} \beta h^{6}}{192} \\
& +\frac{\mathrm{Gr} \beta k h^{5}}{16}
\end{aligned}
$$

Equation (16) gives

$$
\begin{aligned}
& \frac{\partial p}{\partial X} \\
& =\frac{\left(1+\lambda^{\alpha}\left(\partial^{\alpha} / \partial t^{\alpha}\right)\right)(\bar{Q} / \pi)-2 \mathrm{Gr} \beta h^{6} / 96-\operatorname{Gr} \beta k h^{5} / 16}{\left(-h^{4} / 8-k h^{3} / 2\right)} .
\end{aligned}
$$

It is observed that as $\beta \rightarrow 0$ and $k \rightarrow 0$ (12) and (15) reduce to the corresponding result of Tripathi [11].

Pressure rise and friction force at wall are given by

$$
\begin{aligned}
\Delta p & =\int_{0}^{1} \frac{d p}{d X} d X \\
F & =\int_{0}^{1} h^{2}\left(-\frac{d p}{d X}\right) d X .
\end{aligned}
$$

\section{Result and Discussion}

MATHEMATICA package is used to see quantitative effects of various parameters involved in the result on the pumping characteristic and heat transfer. Extensive computation has been performed to reveal the influence of geometry, hydrodynamical parameters on pressure distributation, frictional force, and heat transfer. Parameters analyzed are frictional parameters, nonuniformity of the geometry $B$, amplitude ratio $\emptyset$, material constant $\lambda$, Grashof number $\mathrm{Gr}$, heat source/sink parameter $\beta$, and slip parameter $k$. To discuss the results for the above obtained quantities, the form of the

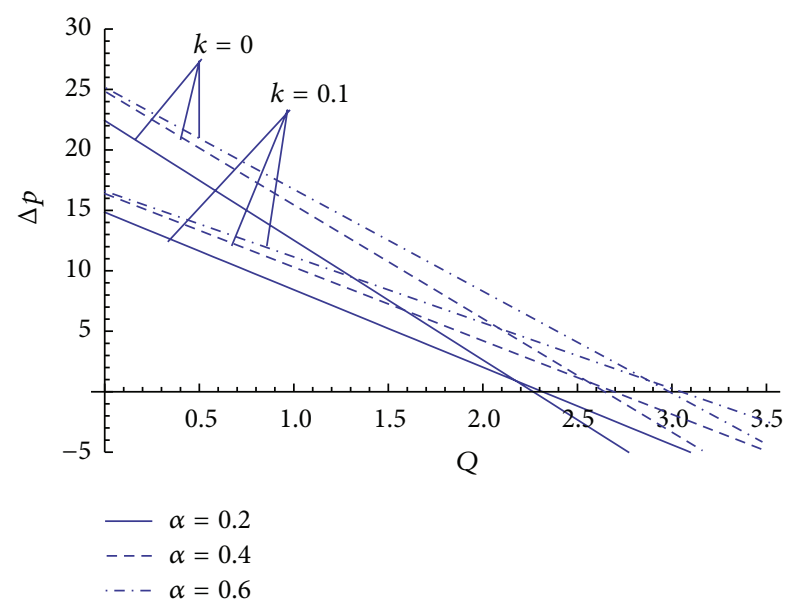

FIGURE 2: Pressure versus averaged flow rate for various values of $\alpha$ at $\lambda=1, B=0.1, t=0.5, \phi=0.4, \mathrm{Gr}=3$, and $\beta=5$.

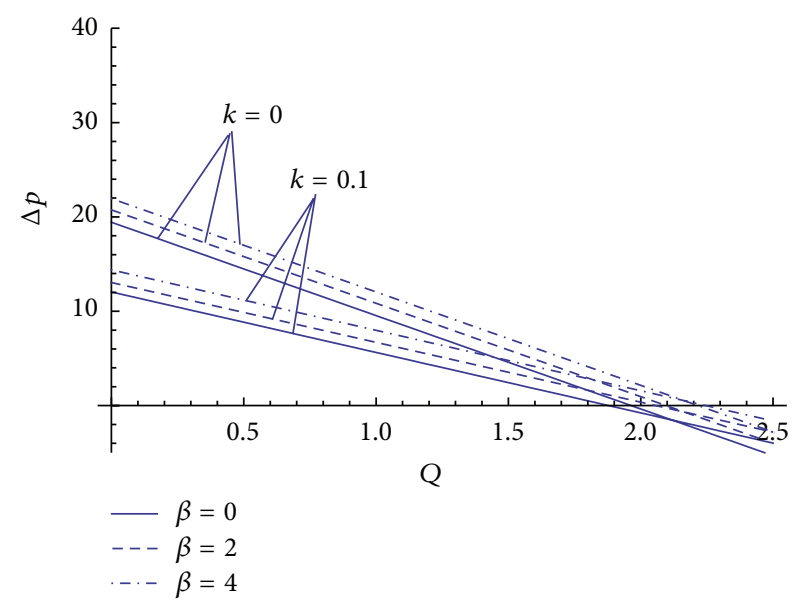

FIGURE 3: Pressure versus averaged flow rate for various values of $\beta$ at $\lambda=1, B=0.1, t=0.5, \phi=0.4, \mathrm{Gr}=3$, and $\alpha=0.2$.

instantaneous volume flow rate $Q(X, t)$, periodic in $(X-t)$, is assumed as follows [17]:

$$
\begin{aligned}
\frac{\bar{Q}(X, t)}{\pi}= & \frac{Q}{\pi}-\frac{\phi^{2}}{2}+\frac{2 B^{\prime} \eta X}{a} \phi \sin 2 \pi(X-t) \\
& +2 \phi \sin 2 \pi(X-t) \\
& +\phi^{2}(\sin 2 \pi(X-t))^{2}
\end{aligned}
$$

where $Q / \pi$ is time average of flow over one period of the wave. The above form of $\bar{Q}(X, t)$ has been assumed in view of the fact that a constant value of $\bar{Q}(X, t)$ gives $\Delta p$ negative, and consequently, there would no pumping action in the tube wall.

Figures 2-7 are plotted to see the variation of pressure distribution for different physical parameters. Figure 2 shows that pressure increases with increase in fractional parameter $\alpha$ for given flow rate. Maximum pressure is obtained at zero flow rate. It is also observed that pressure decreases with increases in slip parameter. Figure 3 reveals the fact that 


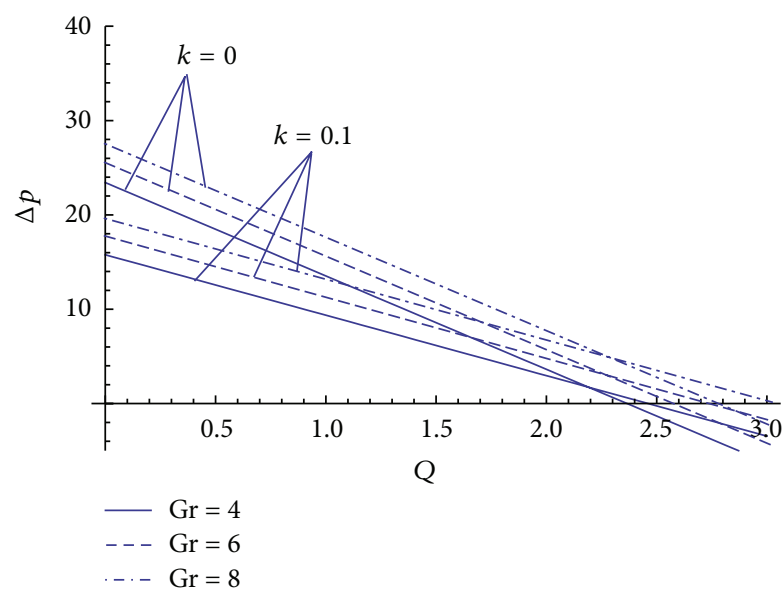

FIGURE 4: Pressure versus averaged flow rate for various values of $\mathrm{Gr}$ at $\lambda=1, B=0.1, t=0.5, \phi=0.4, \beta=5$, and $\alpha=0.2$.

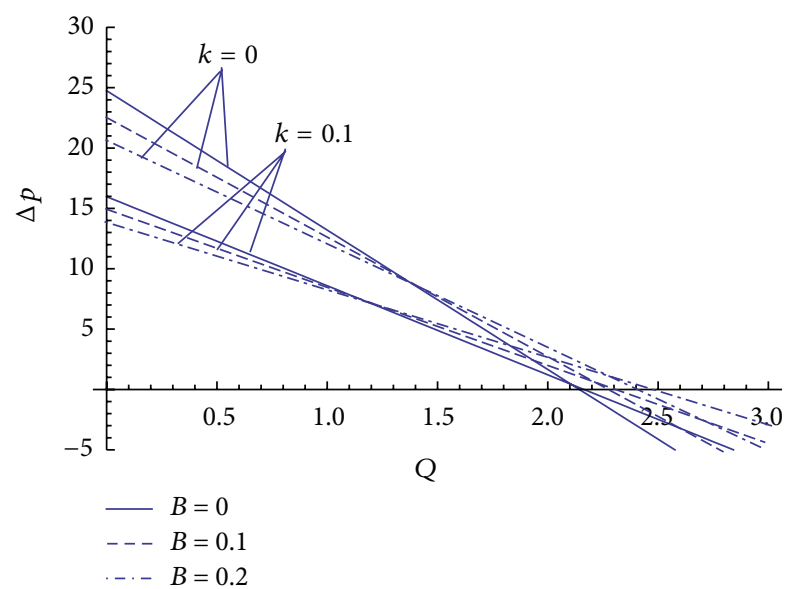

FIGURE 5: Pressure versus averaged flow rate for various values of $B$ at $\lambda=1, \mathrm{Gr}=3, t=0.5, \phi=0.4, \beta=5$, and $\alpha=0.2$.

pressure increases with increase in source/sink parameter $\beta$ for fixed flow rate. For given pressure, flow rate increases with increase in $\beta$ in pumping as well as copumping region. Again maximum pressure is obtained for zero flow rate which is a strong trend of peristaltic fluid dynamics. It can be also seen that pressure decreases with increases in slip parameter. Figure 4 shows the effects of Grashof Number Gr. It is clear from Figure 4 that for given pressure, flow rate increases with increase in Grashof Number Gr in pumping as well as co-pumping region. It can be also seen that pressure increases with increase in Gr for given flow rate. Again, it is observed that pressure decreases with increases in slip parameter. The nonuniformity parameter will exert an important influence on pressure distribution in peristaltic regime. Figure 5 shows the effect of change in nonuniformity parameter $B$ on pressure. It can be seen from Figure 5 that, on increasing the value of $B$, pressure decreases for a given value of flow rate. Pressure decreases with increases in slip parameter at zero flow rate. The variation of pressure against flow rate for various values of amplitude ratio $\emptyset$ is presented

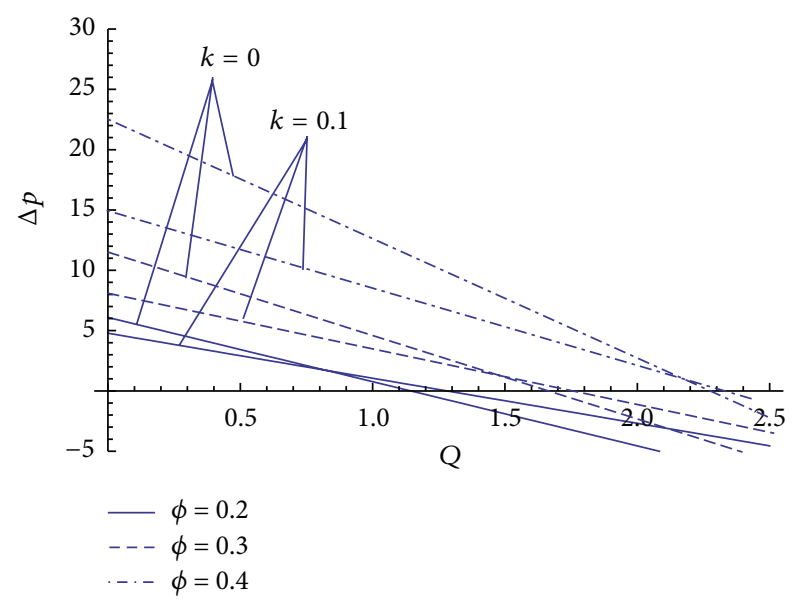

FIgURE 6: Pressure versus averaged flow rate for various values of $\phi$ at $\lambda=1, \mathrm{Gr}=3, t=0.5, B=0.1, \beta=5$, and $\alpha=0.2$.

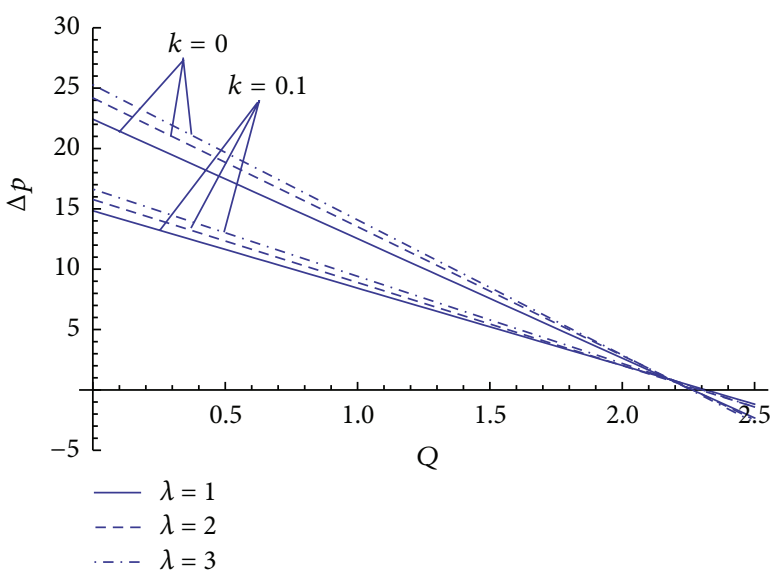

FIGURE 7: Pressure versus averaged flow rate for various values of $\phi \lambda$ at $\phi=0.4, \mathrm{Gr}=3, t=0.5, B=0.1, \beta=5$, and $\alpha=0.2$.

in Figure 6. It is clear from the Figure 6 that, for given flow rate, pressure increases with increases in $\emptyset$. Figure 7 shows the variation of pressure with change in material constant $\lambda$. It is evident from the Figure 7 that, on increasing material constant, pressure also increases. Pressure decreases with increases in slip parameter at zero flow rate.

Figures $8-13$ are plotted to examine the variation of friction force against flow rate for different physical parameters. It is evident from Figures 8-13 that there exist direct proportionality between frictional force $(F)$ and flow rate $(Q)$ irrespective of the parameter varied. Figure 8 shows the variation of frictional force against flow rate with change in fractional parameter $\alpha$. It is clear from the Figure 8 that frictional force decreases with increase in $\alpha$ and it is observed that frictional force is higher with increase in slip parameter at zero flow rate. Figure 9 depicts the variation of frictional force against flow rate for different values of source/sink parameter $\beta$. It can be examined from Figure 9 that frictional force decreases with increase in $\beta$ for given flow rate. The greatest value of friction force is associated with lowest value of $\beta$. Frictional force is higher with increase in slip parameter at 


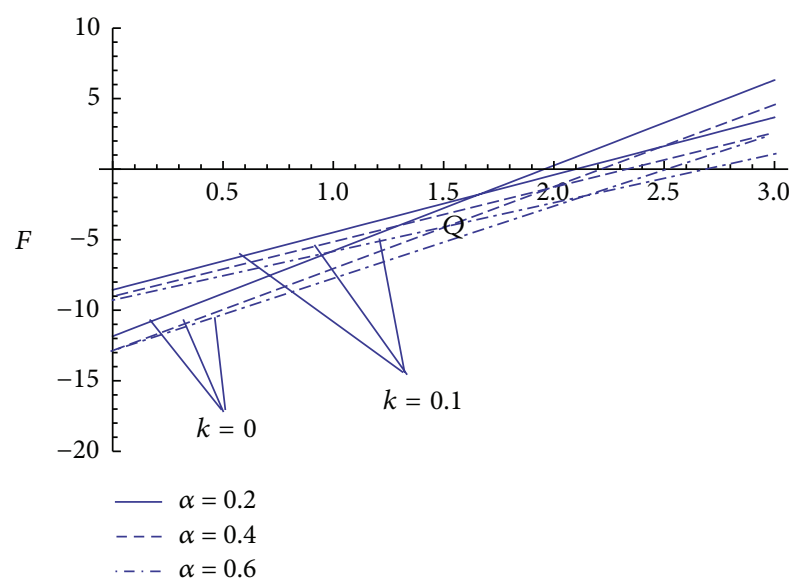

FIGURE 8: Friction force versus averaged flow rate for various values of $\alpha$ at $\lambda=1, t=0.5, \phi=0.4, B=0.1, \mathrm{Gr}=3$, and $\beta=5$.

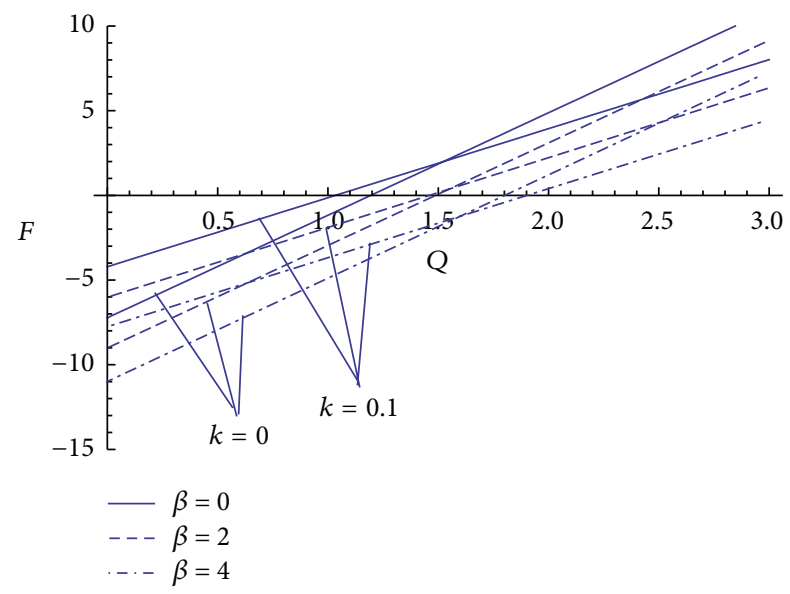

FIGURE 9: Friction force versus averaged flow rate for various values of $\alpha \beta$ at $\lambda=1, t=0.5, \phi=0.4, B=0.1, \mathrm{Gr}=3$, and $\alpha=0.2$.

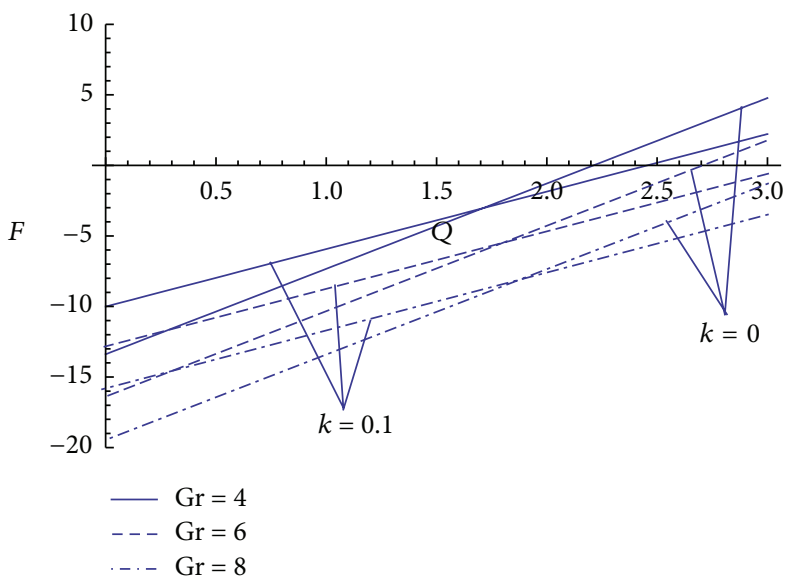

FIGURE 10: Friction force versus averaged flow rate for various values of $\mathrm{Gr}$ at $\lambda=1, t=0.5, \phi=0.4, B=0.1, \alpha=0.2$, and $\beta=5$.

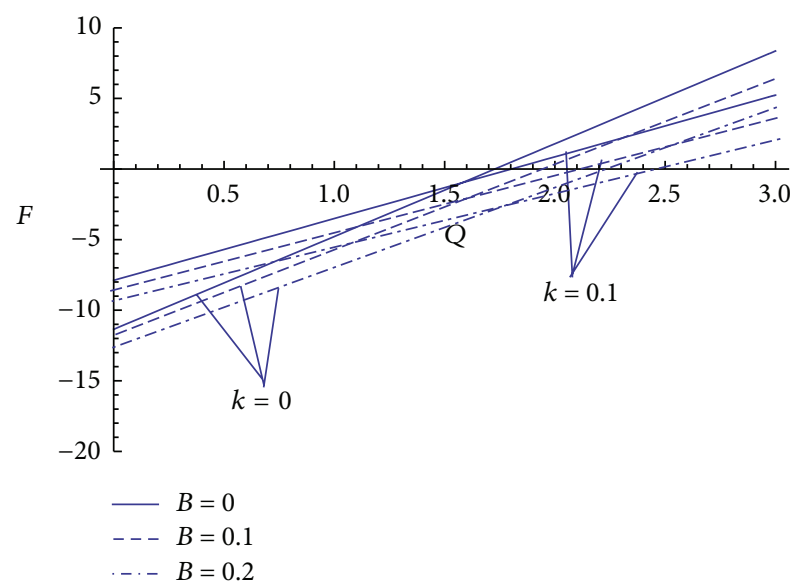

FIGURE 11: Friction force versus averaged flow rate for various values of $B$ at $\lambda=1, t=0.5, \phi=0.4, \mathrm{Gr}=3, \alpha=0.2$, and $\beta=5$.

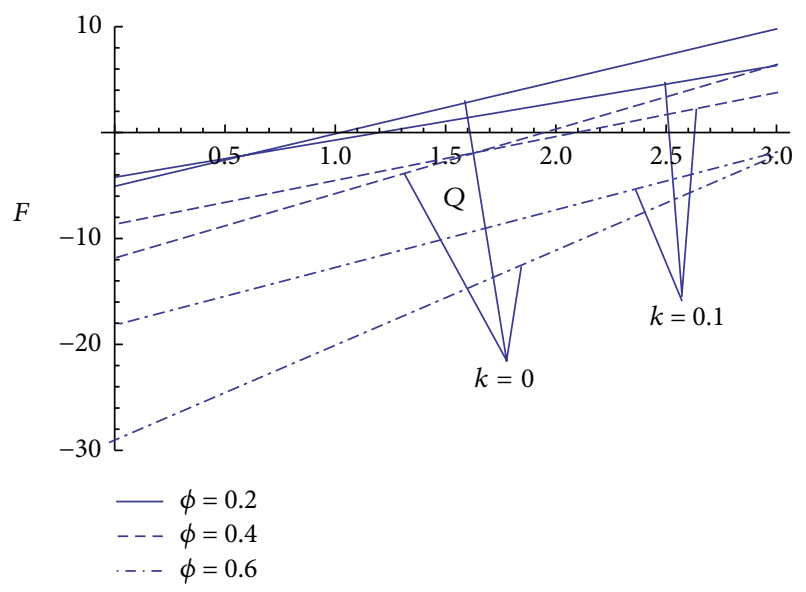

FIGURE 12: Friction force versus averaged flow rate for various values of $\phi$ at $\lambda=1, t=0.5, B=0.1, \mathrm{Gr}=3, \alpha=0.2$, and $\beta=5$.

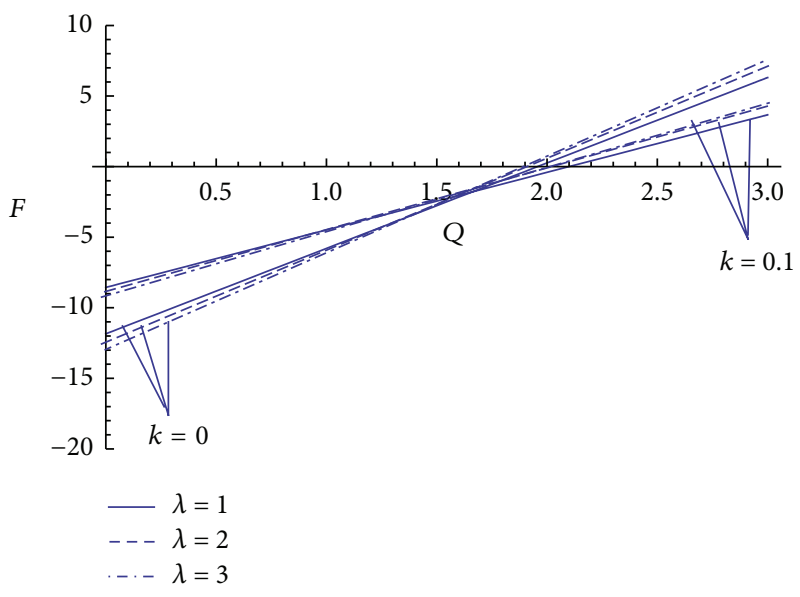

FIGURE 13: Friction force versus averaged flow rate for various values of $\lambda$ at $\phi=0.4, t=0.5, B=0.1, \mathrm{Gr}=3, \alpha=0.2$, and $\beta=5$. 


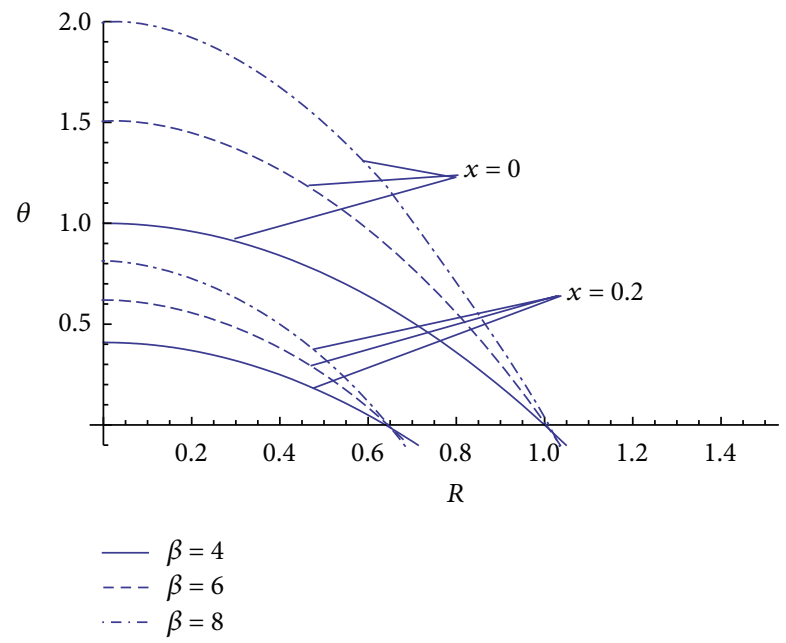

FIgURE 14: Effect of $\beta$ on temperature at $\phi=0.4, B=0.1$, and $t=0.5$ at inlet as well as downstream.

zero flow rate. Figure 10 portrays the variation of frictional force against flow rate for different values of Grashof Number Gr. It is observed from Figure 10 that frictional force decreases with increase in Gr for given flow rate. Frictional force is higher with increase in slip parameter at zero flow rate. It is clear from the Figure 11 that frictional force decreases with increase in $B$. Frictional force is higher with increase in slip parameter at zero flow rate. The effect of amplitude ratio $\emptyset$ on pressure rise is illustrated in Figure 12. It is noted that frictional force decreases with increase in $\emptyset$ for given flow rate. Frictional force is higher with increase in slip parameter at zero flow rate. The effect of material constant $\lambda$ on pressure rise is illustrated in Figure 13. It is evident from Figure 13 that frictional force decreases with increase in $\lambda$. For low flow rate, frictional force is negative but becomes positive for higher flow rate. Frictional force is higher with increase in slip parameter at zero flow rate.

Figures 14-16 show the effects of various parameters on temperature. Temperature increases with increase in source/sink parameter $\beta$ at inlet as well as downstream as portrayed in Figure 14. Temperature is higher at inlet but as we move downstream temperature gradually decreases. Figure 15 illustrates the variation of temperature with amplitude ratio at inlet and downstream. It is clear that temperature decreases with increase in amplitude ratio at inlet and remains constant at downstream. Figure 16 depicts the variation of temperature with nonuniformity parameter $B$. It is clear that temperature remains unaffected with increase in $B$ at inlet but increases downstream. It is also observed that temperature is higher at inlet.

\section{Conclusion}

In this paper, viscoelastic fluid flow with fractional secondgrade model for heat effect with peristaltic transport through nonuniform inclined tube with permeable wall. Exact expression for pressure gradient and temperature are obtained under long wavelength and low Reynolds number. Effects

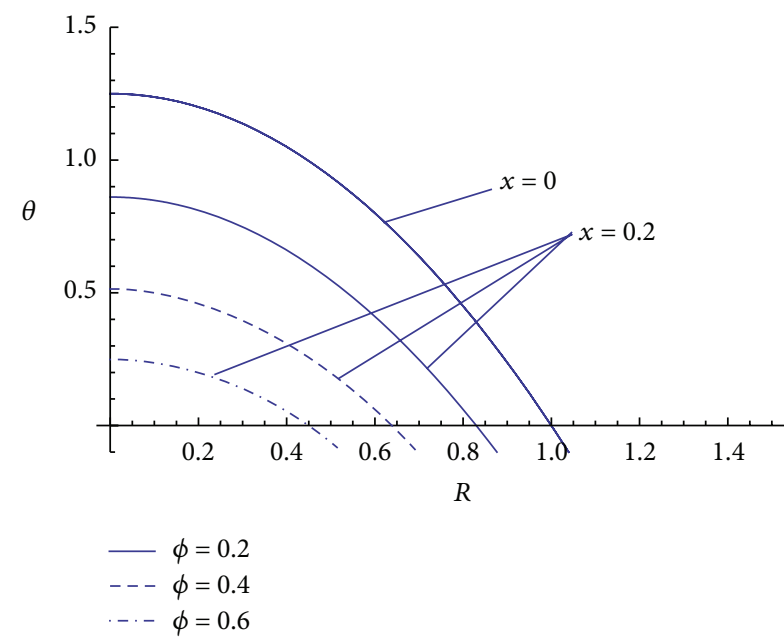

Figure 15: Effect of $\phi$ on temperature at $\beta=5, B=0.1$, and $t=0.5$ at inlet as well as downstream.

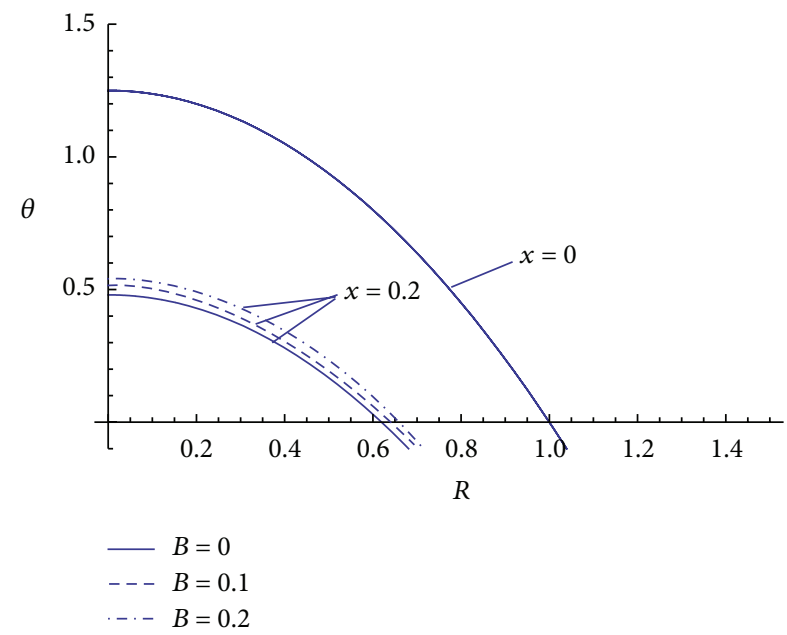

Figure 16: Effect of $B$ on temperature at $\beta=5, \phi=0.4$, and $t=0.5$ at inlet as well as downstream.

of fractional parameter, nonuniformity of tube, permeability of wall, material constant, source/sink parameter, amplitude ratio, and Grashof number are studied. Fractional calculus theory is used to find pressure gradient expression. The following conclusion can be summarized.

(1) The linear relation is found between pressure and flow.

(2) The linear relation is found between friction force and flow.

(3) The pressure function increases with increasing value of fractional parameter, source/sink parameter, Grashof Number, and amplitude ratio.

(4) The variation of friction force against flow rate shows opposite behavior to that of pressure.

(5) Temperature increases with increasing value of source/sink parameter at inlet as well as downstream of the tube. 
(6) Temperature remains constant with increasing value of nonuniformity parameter and amplitude ratio at inlet but may increase with increase in nonuniformity and decreases with increases in amplitude ratio at downstream.

\section{Competing Interests}

The authors declare that they have no competing interests.

\section{References}

[1] W. M. Bayliss and E. H. Starling, "The movement and innervation of the small intestine," The Journal of Physiology, vol. 24, no. 2, pp. 99-143, 1899.

[2] T. W. Latham, Fluid motion in a peristaltic pump [M.S. thesis], Massachusetts Institute of Technology, Cambridge, Mass, USA, 1966.

[3] M. Y. Jaffrin and A. H. Shapiro, "Peristaltic pumping," Annual Review of Fluid Mechanics, vol. 3, pp. 13-36, 1971.

[4] Y. V. K. R. Kumar, P. S. V. H. N. K. Kumari, M. V. R. Murthy, and S. Sreenadh, "Unsteady peristaltic pumping in a finite length tube with permeable wall," Journal of Fluids Engineering, vol. 132, no. 10, Article ID 101201, 4 pages, 2010.

[5] S. A. Victor and V. L. Shah, "Heat transfer to blood flowing in a tube," Biorheology, vol. 12, no. 6, pp. 361-368, 1975.

[6] S. Srinivas and M. Kothandapani, "Peristaltic transport in an asymmetric channel with heat transfer-a note," International Communications in Heat and Mass Transfer, vol. 35, no. 4, pp. 514-522, 2008.

[7] R. Muthuraj and S. Srinivas, "Mixed convective heat and mass transfer in a vertical wavy channel with traveling thermal waves and porous medium," Computers \& Mathematics with Applications, vol. 59, no. 11, pp. 3516-3528, 2010.

[8] G. Radhakrishnamacharya and V. Radhakrishna, "Heat transfer to peristaltic transport in a non-uniform channel," Defence Science Journal, vol. 43, no. 3, pp. 275-280, 1993.

[9] R. Ellahi, M. M. Bhatti, and K. Vafai, "Effects of heat and mass transfer on peristaltic flow in a non-uniform rectangular duct," International Journal of Heat and Mass Transfer, vol. 71, pp. 706719, 2014.

[10] H. Qi and M. Xu, "Unsteady flow of viscoelastic fluid with fractional Maxwell model in a channel," Mechanics Research Communications, vol. 34, no. 2, pp. 210-212, 2007.

[11] D. Tripathi, "Peristaltic flow of a fractional second grade fluid through a cylindrical tube," Thermal Science, vol. 15, supplement 2, pp. 167-173, 2011.

[12] D. Tripathi and O. A. Bég, "Peristaltic propulsion of generalized Burgers' fluids through a non-uniform porous medium: a study of chyme dynamics through the diseased intestine," Mathematical Biosciences, vol. 248, pp. 67-77, 2014.

[13] M. Hameed, A. A. Khan, R. Ellahi, and M. Raza, "Study of magnetic and heat transfer on the peristaltic transport of a fractional second grade fluid in a vertical tube," Engineering Science and Technology, an International Journal, vol. 18, no. 3, pp. 496-502, 2015.

[14] V. P. Rathod and A. Tuljappa, "Peristaltic flow of fractional second grade fluid through a cylindricakl tube with heat transfer," Journal of Chemical, Biological and Physical Sciences, vol. 5, pp. 1841-1855, 2015.
[15] V. K. Narla, K. M. Prasad, and J. V. Ramanamurthy, "Peristaltic motion of viscoelastic fluid with fractional second grade model in curved channels," Chinese Journal of Engineering, vol. 2013, Article ID 582390, 7 pages, 2013.

[16] P. G. Saffman, "On the boundary condition at the surface of a porous medium," Studies in Applied Mathematics, vol. 50, no. 2, pp. 93-101, 1971.

[17] L. M. Srivastava and V. P. Srivastava, "Peristaltic transport of a power-law fluid: application to the ductus efferentes of the reproductive tract," Rheologica Acta, vol. 27, no. 4, pp. 428-433, 1988. 

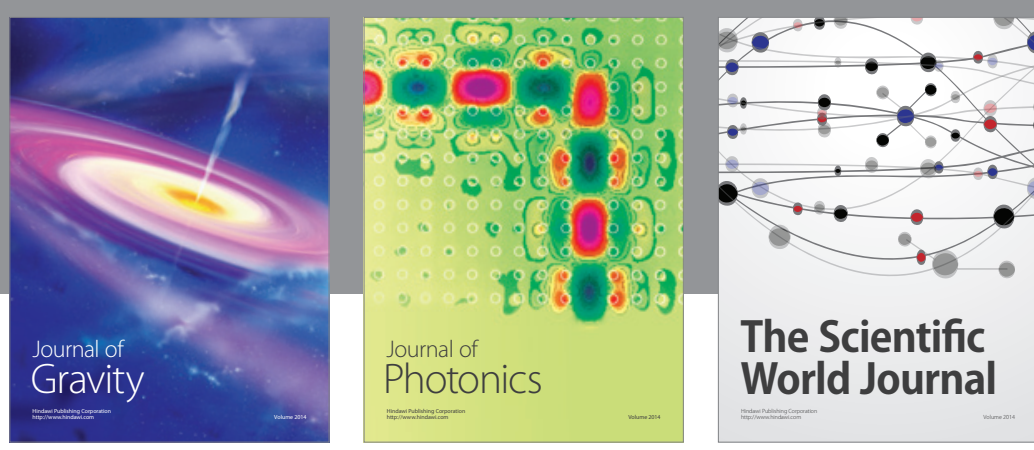

The Scientific World Journal
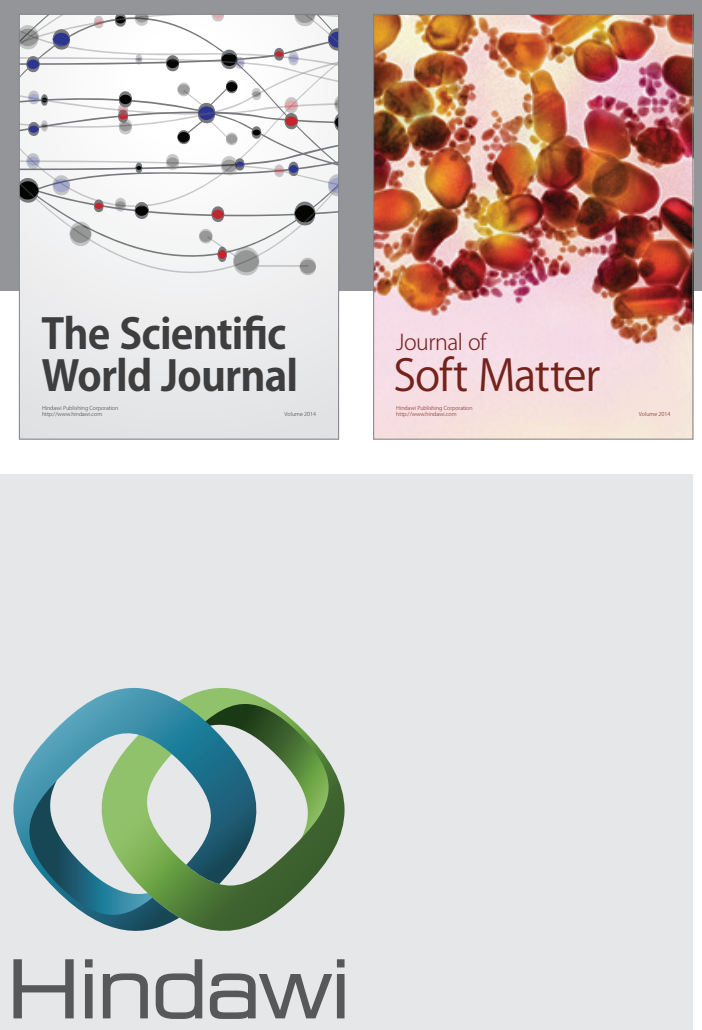

Submit your manuscripts at

http://www.hindawi.com

nternational Journal of

Statistical Mechanics
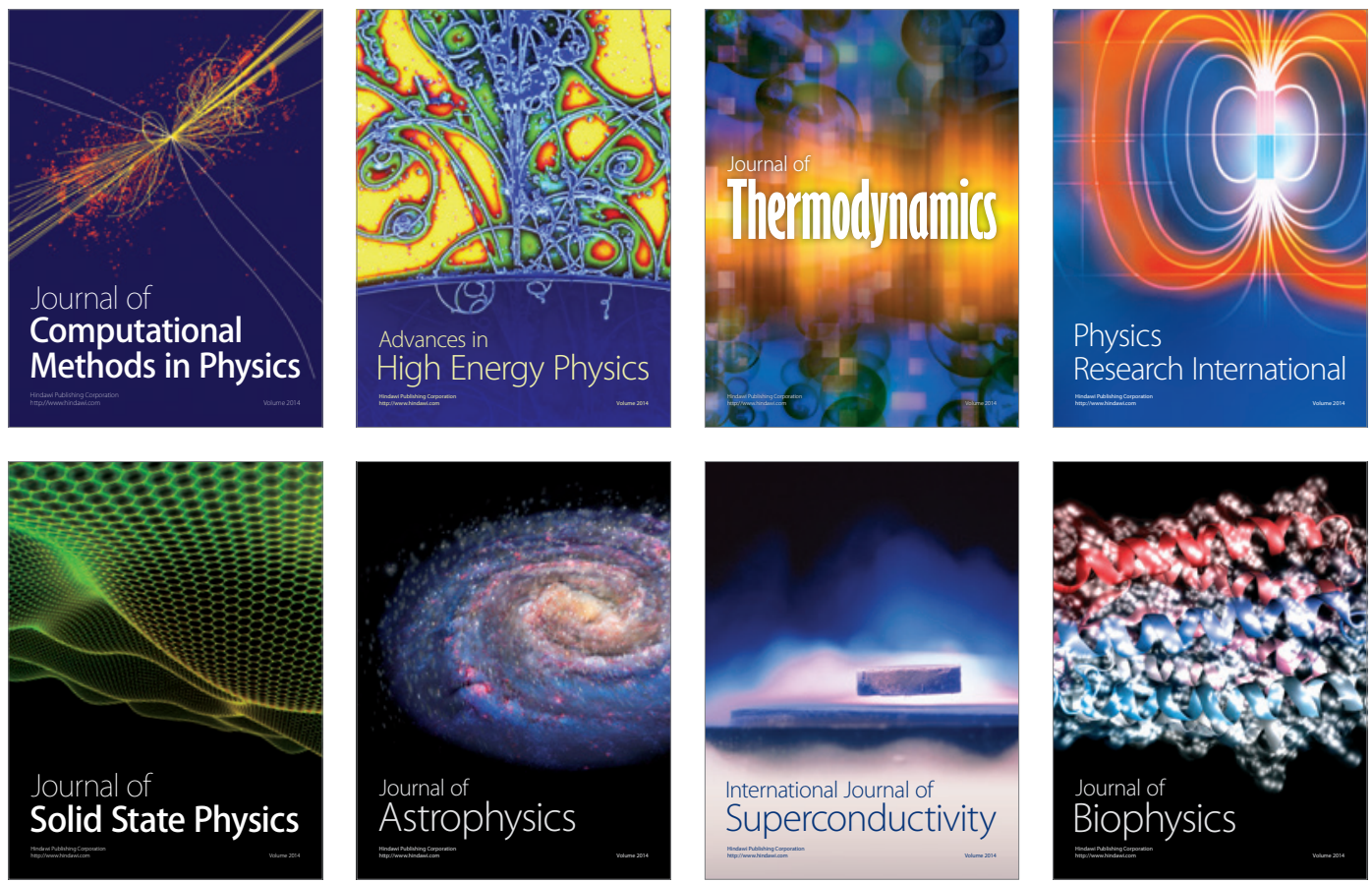
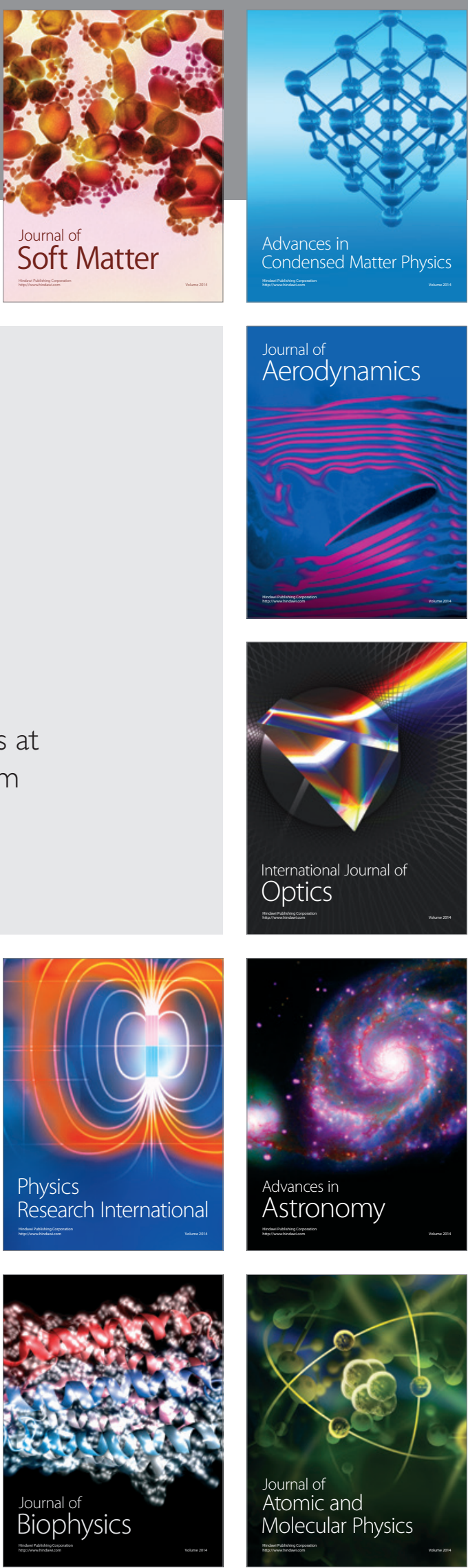